\title{
Reclassification of Shigella species as later heterotypic synonyms of Escherichia coli in the Genome Taxonomy Database
}

\author{
Donovan H. Parks, Maria Chuvochina, Peter R. Reeves, Scott A. Beatson, Philip Hugenholtz
}

Members of the genus Shigella have high genomic similarity to Escherichia coli and are often considered to be atypical members of this species. In an attempt to retain Shigella species as recognizable entities, they were reclassified as Escherichia species in the Genome Taxonomy Database (GTDB) using an operational average nucleotide identity (ANI)-based approach nucleated around type strains. This resulted in nearly $80 \%$ of $E$. coli genomes being reclassified to new species including the common laboratory strain $E$. coli K-12 (to ' $E$. flexneri') because it is more closely related to the type strain of Shigella flexneri than it is to the type strain of $E$. coli. Here we resolve this conundrum by treating Shigella species as later heterotypic synonyms of $E$. coli, present evidence supporting this reclassification, and show that assigning E. coli/Shigella strains to a single species is congruent with the GTDB-adopted genomic species definition.

\section{Introduction}

The genus Escherichia currently comprises the six species E. coli (Castellani and Chalmers, 1919), E. hermannii (Brenner et al., 1982), E. fergusonii (Farmer et al., 1985), E. albertii (Huys et al., 2003), E. marmotae (Liu et al., 2015), and the recently described E. ruysiae (van der Putten et al., 2021). However, a recent phylogenetic study places $E$. hermannii within the effectively published but currently unvalidated genus 'Atlantibacter' (Hata et al., 2016), and both the NCBI (Schoch et al., 2020) and GTDB (Parks et al., 2018) taxonomies follow this reclassification. There are also a number of strains that have been recognized as distinct Escherichia lineages that have no assigned species name and are referred to as 'cryptic clades' (Walk et al., 2009). However, the most enduring anomaly with this genus is paraphyly between E. coli and the four currently recognized Shigella species (The et al. 2016; Pettengill et al., 2016; Hu et al., 2019), which has been attributed to independent acquisition of the pINV virulence plasmid and subsequent niche adaptation (Pupo et al., 2000; Lan and Reeves 2002; Yang et al., 2005).

Numerous studies have shown that $E$. coli and Shigella species have high genomic similarity (Brenner et al., 1973; Richter and Rosselló-Móra 2009; Jain et al., 2018; Ciufo et al., 2018). Consequently, it is widely recognized that E. coli and Shigella species could be considered a single species (Lan and Reeves, 2002; Richter and Rosselló-Móra, 2009; van den Beld and Reubsaet, 2012); however, this reclassification has not been adopted due to the medical significance of Shigella as pathogens causing shigellosis, a form of bacillary dysentery (Ewing 1949; Sahl et al., 2015). At the same time, enteroinvasive E. coli (EIEC) also causes dysentery making the clinical distinction between E. coli and Shigella ambiguous as few biochemical properties distinguish Shigella from EIEC (Johnson 2000; Yang et al., 2005; van den Beld 2012; Hendriks et al., 2020). Studies have also shown that E. coli and Shigella species are paraphyletic and that S. boydii, S. dysenteriae, and S. flexneri, primarily defined based on serotype, are not 
monophyletic entities (Sahl et al., 2015; Pettengill et al., 2016; Pupo et al., 2000; Hu et al., 2019) further justifying the reclassification of Shigella species as E. coli.

The Genome Taxonomy Database (GTDB) has adopted a genomic species definition based on average nucleotide identity (ANI) and alignment fraction (AF) nucleated around genomes from type strains for delineating species (Parks et al., 2020) and an estimation of time of divergence for circumscribing higher taxonomic ranks (Parks et al., 2018). Application of these criteria resulted in Shigella species being reassigned to the genus Escherichia as 'E. flexneri' and 'E. dysenteriae' with S. sonnei and S. boydii being classified as synonyms of 'E. flexneri' due to their high genomic relatedness (>97\%). Notably, nearly $80 \%$ of the genomes in GTDB R06-RS202 formerly classified as E. coli were reclassified as either ' $E$. flexneri' or 'E. dysenteriae' as a result of the adopted criteria for delineating species. This includes the common laboratory strain E. coli str. K-12 which was assigned to 'E. flexneri' due to its higher genomic similarity to the type strain of 'E. flexneri' than to the type strain of E. coli (U5/41 = ATCC 11775; Meier-Kolthoff et al., 2014).

A practical consequence of these GTDB reassignments is that traditional properties of species such as ' $E$. dysenteriae' and 'E. flexneri' being comprised of human pathogenic strains are no longer applicable. As this is likely to result in confusion, we propose that Shigella spp. be treated as later heterotypic synonyms of $E$. coli in GTDB. Here we put forth the evidence supporting this reclassification in anticipation of introducing this change in the next release of GTDB.

\section{Results and discussion}

\section{Reclassification of Escherichia coli and Shigella in GTDB}

GTDB assigns strains to species based on the ANI and AF to genomes assembled from the type strain of the species (henceforth referred to as type strain genomes) or to a selected representative genome of the species when a type strain genome is not available (Parks et al., 2020). This provides quantitative criteria for establishing species membership that is consistent with the majority of species assignments defined using a polyphasic approach. In GTDB release R06-RS202, 68.2\% of genomes with an NCBI species assignment have the same species assignment in the GTDB. Genomes with incongruent GTDB and $\mathrm{NCBI}$ species assignments result from the transfer of species to other genera (10.4\%), defining overclassified species as synonyms (1.4\%), dividing species considered to be underclassified into multiple species (5.9\%), and otherwise reclassifying genomes consider misclassified according to the GTDB (14.1\%; Supp. Table 1). Notably, $>50 \%$ of incongruent species assignments arise from just nine NCBI species with E. coli and Shigella spp. being the most conspicuous accounting for $32.6 \%$ of all incongruent assignments between GTDB and NCBI (Table 1; Supp. Table 2). This is in contrast to other Escherichia species where all or nearly all genomes assigned to these species within GTDB have the same assignment in the NCBI Taxonomy (Table 1).

Strains have traditionally been assigned to E. coli and Shigella spp. based on biochemistry and serotyping (Pettengill et al., 2016; Chattaway et al., 2017) which is in contrast to the genomic species definition (Doolittle and Papke 2006; Konstantinidis et al., 2006; Richter and Rosselló-Móra, 2009) adopted by GTDB. Specifically, GTDB delineates species using a fixed AF threshold of 0.65 and an ANI threshold that 
is allowed to vary between $95 \%$ and $97 \%$ in order to preserve the majority of existing species classifications (Parks et al., 2020). Despite using a flexible ANI threshold, 'E. sonnei' and 'E. boydii' are considered later heterotypic synonyms of 'E. flexneri' in GTDB as the ANI between these type strain genomes is $97.9 \%$ and $97.8 \%$, respectively (Fig. 1). Furthermore, $79.2 \%$ of the 20,973 genomes classified as $E$. coli at NCBI are reassigned within GTDB with the majority being reclassified as ' $E$. flexneri' $(12,400$ genomes) or ' $E$. dysenteriae' (2,044 genomes; Table 1$)$. Similarly, the majority (68.3\%) of genomes classified as $S$. dysenteriae at NCBI are classified as ' $E$. flexneri' in GTDB (Table 1). GTDB species assignments reflect the closest type strain genome as determined using ANI and this large number of reassignments illustrate the degree to which the traditional classification of E. coli and Shigella spp. conflict with the adopted genomic species definition (Fig. 1). A few clear misclassifications within the $\mathrm{NCBI}$ taxonomy are also evident such as the reassignment of a $S$. sonnei and three $S$. boydii genomes to species in the genus Serratia in GTDB (Supp. Table 3).

Table 1. GTDB R06-RS202 assignment of genomes classified as Escherichia or Shigella within the NCBI taxonomy.

\begin{tabular}{|c|c|c|c|c|}
\hline NCBI species & $\begin{array}{l}\text { No. } \\
\text { genomes }\end{array}$ & $\begin{array}{l}\text { No. reassigned } \\
\text { genomes }\end{array}$ & $\begin{array}{l}\text { Reassigned } \\
\text { genomes (\%) }\end{array}$ & GTDB species \\
\hline Escherichia coli & 20,973 & $16,609(79.19 \%)$ & 28.28 & $\begin{array}{l}\text { E. flexneri: } 12,400 \text { (59.12\%); E. coli: 4,364 (20.81\%); E. coli_D: } \\
\text { 2,094 (9.98\%); E. dysenteriae: 2,044 (9.75\%); E. coli_C: } 45 \\
\text { (0.21\%); E. sp000208585: } 9 \text { (0.04\%); E. sp005843885: } 2(0.01 \%) ; \\
\text { Klebsiella quasipneumoniae: } 1(0.00 \%) ; \text { E. coli_E: } 1(0.00 \%) ; \\
\text { Phytobacter ursingii: } 1 \text { (0.00\%); Citrobacter freundii: } 1 \text { (0.00\%); } \\
\text { Enterobacter roggenkampii: } 1 \text { (0.00\%); Pseudomonas_E } \\
\text { sp002113295: } 1 \text { (0.00\%); E. albertii: } 1(0.00 \%) ; \text { Kluyvera } \\
\text { georgiana_A: } 1 \text { (0.00\%); Hafnia alvei: } 1 \text { (0.00\%); E. marmotae: } 1 \\
\text { (0.00\%);Citrobacter gillenii: } 1 \text { (0.00\%); Providencia rettgeri_D: } 1 \\
\text { (0.00\%); Enterobacter hormaechei_A: } 1(0.00 \%) ; \text { Klebsiella } \\
\text { aerogenes: } 1(0.00 \%) ; \text { Klebsiella variicola: } 1(0.00 \%)\end{array}$ \\
\hline Shigella sonnei & 1,368 & $1,368(100.00 \%)$ & 2.33 & $\begin{array}{l}\text { E. flexneri: 1,354 (98.98\%); E. coli_D: } 13 \text { (0.95\%); Serratia } \\
\text { marcescens_K: } 1(0.07 \%)\end{array}$ \\
\hline Shigella flexneri & 706 & $706(100.00 \%)$ & 1.20 & E. flexneri: 705 (99.86\%); E. coli: 1 (0.14\%) \\
\hline Shigella boydii & 120 & $120(100.00 \%)$ & 0.20 & $\begin{array}{l}\text { E. flexneri: } 111(92.50 \%) \text {; E. coli_D: } 5(4.17 \%) \text {; Serratia } \\
\text { marcescens_I: } 3(2.50 \%) ; \text { E. dysenteriae: } 1(0.83 \%)\end{array}$ \\
\hline Shigella dysenteriae & 60 & $60(100.00 \%)$ & 0.10 & $\begin{array}{l}\text { E. flexneri: } 41 \text { (68.33\%); E. dysenteriae: } 18(30.00 \%) ; \text { E. coli_D: } 1 \\
(1.67 \%)\end{array}$ \\
\hline Escherichia fergusonii & 74 & $1(1.35 \%)$ & 0.00 & E. fergusonii: 73 (98.65\%); E. flexneri: 1 (1.35\%) \\
\hline Escherichia albertii & 98 & $0(0.00 \%)$ & 0.00 & E. albertii: 98 (100.00\%) \\
\hline Escherichia marmotae & 34 & $0(0.00 \%)$ & 0.00 & E. marmotae: $34(100.00 \%)$ \\
\hline Escherichia ruysiae & $\mathrm{n} / \mathrm{a}$ & $\mathrm{n} / \mathrm{a}$ & $\mathrm{n} / \mathrm{a}$ & (proposed after GTDB R06-RS202; see Methods) \\
\hline $\begin{array}{l}\text { Unclassified } \\
\text { Escherichia sp. }\end{array}$ & 153 & $153(100.00 \%)$ & 0.26 & $\begin{array}{l}\text { E. marmotae: } 42 \text { (27.45\%); E. sp005843885: } 35 \text { (22.88\%); E. } \\
\text { sp000208585: } 22 \text { (14.38\%); E. flexneri: } 18 \text { (11.76\%); E. coli_C: } 17 \\
\text { (11.11\%); E. coli_D: } 8 \text { (5.23\%); E. coli: } 3 \text { (1.96\%); E. sp004211955: } \\
2 \text { (1.31\%); E. sp001660175: } 2 \text { (1.31\%); Citrobacter freundii: } 1 \\
\text { (0.65\%); E. sp002965065: } 1 \text { (0.65\%); PseudE. vulneris: } 1 \text { (0.65\%); } \\
\text { PseudE. sp002918705: } 1 \text { (0.65\%) }\end{array}$ \\
\hline $\begin{array}{l}\text { Unclassified } \\
\text { Shigella sp. }\end{array}$ & 112 & $112(100.00 \%)$ & 0.19 & $\begin{array}{l}\text { E. flexneri: } 107 \text { (95.54\%); Proteus sp001722135: } 2 \text { (1.79\%); E. } \\
\text { coli_D: } 2 \text { (1.79\%); E. coli: } 1(0.89 \%)\end{array}$ \\
\hline
\end{tabular}


A

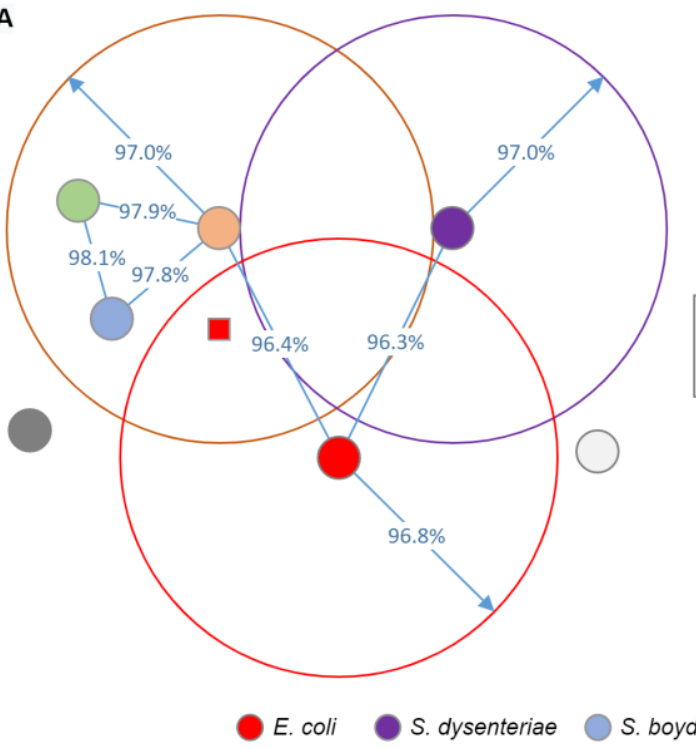

B

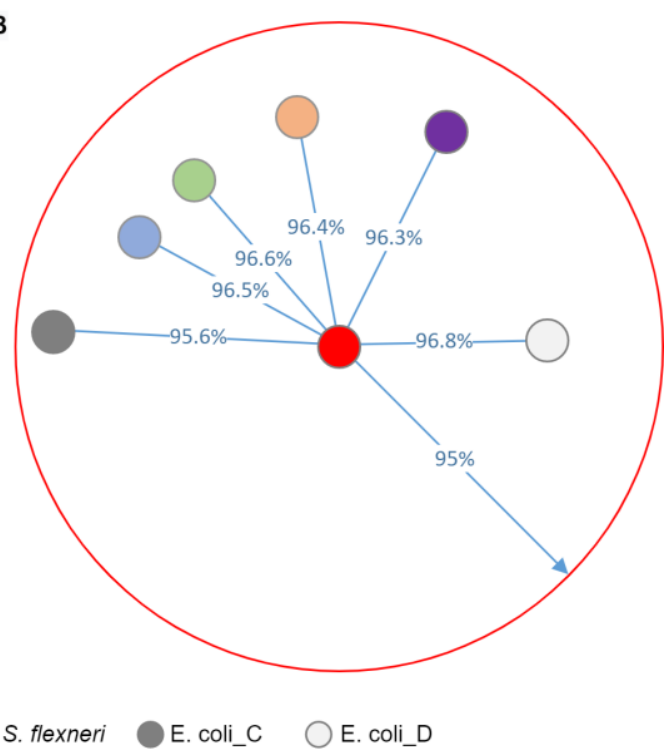

Figure 1. Conceptual diagram showing the high genomic similarity between Escherichia and Shigella species (A), and the proposal to reclassify Shigella spp. as later heterotypic synonyms of $E$. coli (B). Filled circles indicate genomes assembled from the type strain of E. coli or a Shigella spp., and the representative genomes from the GTDB species E. coli_C and E. coli_D. The distance between genomes indicates their ANI though the diagram is conceptual as it is not possible to accurately represent the pairwise distance between all genomes. The larger circles depict the ANI criteria used by GTDB for assigning genomes to each species (Parks et al., 2020). This criterion is typically 95\% in the GTDB, but the high similarity of these species results in this criterion being increased. The single red square represents a genome traditionally classified as $E$. coli which is reclassified as $S$. flexneri in GTDB since it resides within the ANI threshold of both species, but is more similar to the $S$. flexneri type strain genome. Reclassification of Shigella spp. as later heterotypic synonyms of $E$. coli results in a species with a $95 \%$ ANI criterion commensurate with the majority of GTDB species. E. coli_C and E. coli_D are also reclassified as E. coli as they have an ANI $>95 \%$ to the type strain of $E$. coli.

\section{Genomes assembled from type strains of a species are highly similar}

Here we verify that type strain genomes from Escherichia and Shigella species are highly similar to each other giving confidence that the assemblies are of high quality and unlikely to be misclassified (Table 2). All species except $E$. albertii, E. ruysiae, and S. flexneri had two or more type strain genomes available, and intraspecific type strain genomes had an ANI $>99.8 \%$ with an AF generally $>0.97$. Lower AFs were observed in a few cases, but this can be attributed to either assembly quality (e.g., E. marmotae assembly GCF_000807695.1 consists of 218 contigs compared to the single chromosome and two plasmids of the E. marmotae assembly GCF_002900365.1) or naturally occurring differences in the phage or genomic islands within individual genomes. 
Table 2. Comparison of genomes assembled from type strains of Escherichia and Shigella species.

\begin{tabular}{|c|c|c|c|c|c|c|}
\hline Type strain & $\begin{array}{l}\text { No. type } \\
\text { genomes }\end{array}$ & $\begin{array}{c}\text { Mean } \\
\text { ANI }\end{array}$ & $\begin{array}{c}\text { Mean } \\
\boldsymbol{A F}\end{array}$ & $\begin{array}{c}\text { Minimum } \\
\text { ANI }\end{array}$ & $\begin{array}{c}\text { Minimum } \\
\qquad F\end{array}$ & Type strain genomes ${ }^{\#}$ \\
\hline Escherichia albertii NBRC 107761 & 1 & $\mathrm{n} / \mathrm{a}$ & $\mathrm{n} / \mathrm{a}$ & $\mathrm{n} / \mathrm{a}$ & $\mathrm{n} / \mathrm{a}$ & GCF_000759775.1 \\
\hline Escherichia coli ATCC 11775 & 5 & 99.92 & 0.982 & 99.83 & 0.973 & $\begin{array}{l}\text { GCF_003697165.2, GCA_900706755.1, } \\
\text { GCF_000734955.1, GCF_000690815.1, } \\
\text { GCA_000613265.1 }\end{array}$ \\
\hline Escherichia fergusonii NCTC 12128 & 2 & 99.99 & 0.998 & 99.99 & 0.998 & GCF_000026225.1, GCF_900450565.1 \\
\hline Escherichia marmotae HT 073016 & 2 & 99.94 & 0.939 & 99.94 & 0.939 & GCF_002900365.1, GCF_000807695.1 \\
\hline Escherichia ruysiae OРT1704 & 1 & $\mathrm{n} / \mathrm{a}$ & $\mathrm{n} / \mathrm{a}$ & $\mathrm{n} / \mathrm{a}$ & $\mathrm{n} / \mathrm{a}$ & GCF_902498915.1 \\
\hline Shigella boydii ATCC 8700 & 2 & 99.97 & 0.951 & 99.97 & 0.951 & GCF_002946735.1, GCA_900457095.1 \\
\hline Shigella dysenteriae NCTC 4837 & 2 & 100.0 & 0.979 & 100.00 & 0.979 & GCF_002949675.1, GCF_900457215.1 \\
\hline Shigella flexneri ATCC 29903 & 1 & $\mathrm{n} / \mathrm{a}$ & $\mathrm{n} / \mathrm{a}$ & $\mathrm{n} / \mathrm{a}$ & $\mathrm{n} / \mathrm{a}$ & GCF_002950215.1 \\
\hline Shigella sonnei NCTC 12984 & 2 & 100.0 & 0.991 & 100.00 & 0.991 & GCA_002950395.1, GCF_900457155.1 \\
\hline
\end{tabular}

\# genomes in bold used in subsequent analyses involving type strain genomes

\section{Phylogeny and genomic similarity of Escherichia and Shigella type strains}

A maximum-likelihood tree was used to establish the phylogenetic relationships between type strain genomes for Escherichia and Shigella species along with the representative genomes of the seven GTDB R06-RS202 placeholder species within Escherichia (Fig. 2A; Supp. Table 4). As expected, E. coli and Shigella spp. form a monophyletic lineage which also includes the GTDB species E. coli_D. Genomic species are generally defined as strains with an ANI greater than $94 \%$ to $96 \%$ and an AF greater than 0.5 to 0.65 (Kostantinidis and Tiedje, 2005; Goris et al., 2007; Varghese et al., 2015; Ciufo et al., 2018; Jain et al., 2019). The type strain genomes for E. coli and the Shigella spp. along with the representative genomes for E. coli_D and E. coli_C satisfy these criteria with the most dissimilar species being $S$. dysenteriae and E. coli_C at 95.3\% ANI and 0.81 AF (Fig. 2B; Supp. Table 5). Furthermore, all genomes classified as E. coli, 'E. flexneri', 'E. dysenteriae', E. coli_C, or E. coli_D in GTDB have an ANI $\geq 95 \%$ and AF $\geq 0.65$ to the E. coli type strain (ATCC $11775^{\top}$ ) genome when using FastANI (Figs. 2C and 2D), and thus satisfy the GTDB criteria for classifying these strains as a single species (Parks et al., 2020). This result is robust to the method used to establish genomic similarity as indicated by BLAST-based ANI (Rodriguez-R and Konstantinidis, 2016) providing highly correlated results with all E. coli, 'E. flexneri', 'E. dysenteriae', E. coli_C, and E. coli_D genomes still reported as having $\geq 95 \%$ ANI to E. coli ATCC $11775^{\top}$ (Supp. Fig. 1). 
bioRxiv preprint doi: https://doi.org/10.1101/2021.09.22.461432; this version posted September 22, 2021. The copyright holder for this preprint (which was not certified by peer review) is the author/funder, who has granted bioRxiv a license to display the preprint in perpetuity. It is made available under aCC-BY 4.0 International license.

(A)

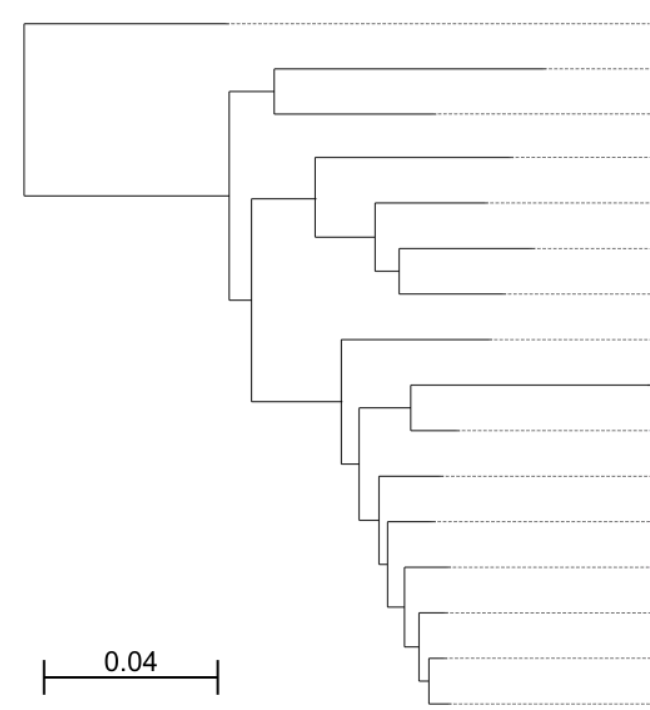

(B)

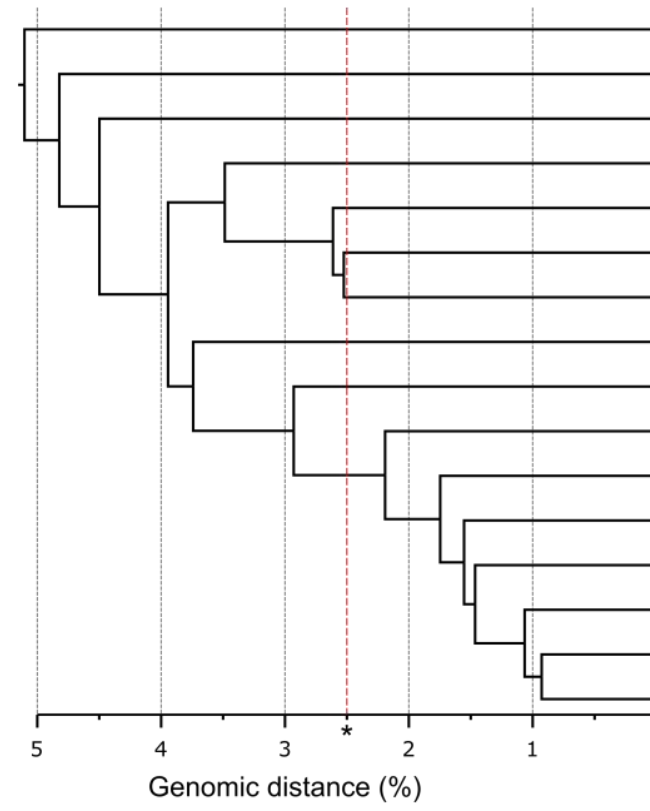

GTDB Species

E. albertii

E. marmotae

E. ruysiae

E. coli_E

E. sp005843885

E. sp001660175

E. sp004211955

E. sp002965065

E. fergusonii

E. coli_C

E. coli

E. coli_D

E. dysenteriae

E. flexneri

E. flexneri

E. flexneri

GTDB Species

E. albertii

E. fergusonii

E. marmotae

E. coli_E

E. sp005843885

E. sp001660175

E. sp004211955

E. ruysiae

E. sp002965065

E. coli_C

E. coli

E. coli_D

E. dysenteriae

E. flexneri

E. flexneri

E. flexneri

* equivalent to $95 \% \mathrm{ANI}$
NCBI Species

E. albertii

E. marmotae

E. ruysiae

E. coli

unclassified

unclassified

unclassified

unclassified

E. fergusonii

E. coli

E. coli

E. coli

S. dysenteriae

S. flexneri

S. sonnei

S. boydii

\section{NCBI Species}

E. albertii

E. fergusonii

E. marmotae

E. coli

unclassified

unclassified

unclassified

E. ruysiae

unclassified

E. coli

E. coli

E. coli

S. dysenteriae

S. flexneri

S. sonnei

S. boydii
Strain ID

NBRC 107761

HT073016

OPT1704

SCPM-O-B-8794

E4742

B1147

E1V33

MOD1-EC7003

ATCC 35469

2013C-4282

ATCC 11775

UMN026

ATCC 13313

ATCC 29903

ATCC 29930

ATCC 8700

Strain ID

NBRC 107761

ATCC 35469

HT073016

SCPM-O-B-8794

E4742

B1147

E1V33

OPT1704

MOD1-EC7003

2013C-4282

ATCC 11775

UMN026

ATCC 13313

ATCC 29903

ATCC 29930

ATCC 8700

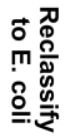

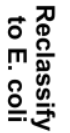

(C)
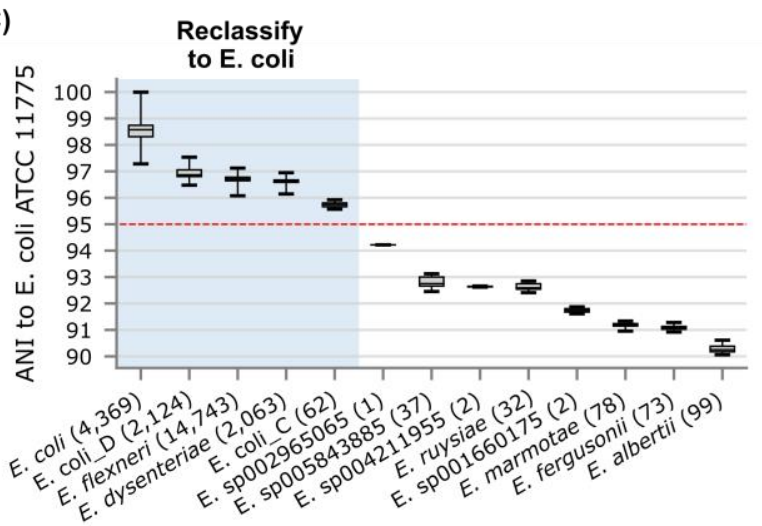

(D)
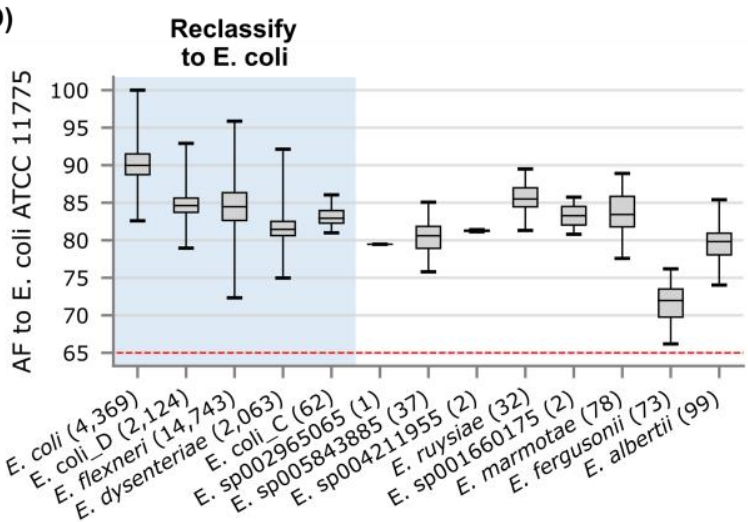
Figure 2. Phylogenetic and genomic similarity of Escherichia and Shigella species. (A) Phylogenetic tree inferred from the concatenated multiple sequence alignment of 2,329 core genes using IQ-Tree under the GTR+F+R5 model. E. albertii NBRC $107761^{\top}$ was used to root the tree (see Methods). SH-aLRT and UFBoot support values were $100 \%$ for all nodes. (B) UPGMA tree indicating the genomic distance, i.e. 100-ANI, between Escherichia and Shigella type strain genomes as determined with FastANI. (C) ANI between the E. coli ATCC $11775^{\top}$ genome and all genomes classified as Escherichia in GTDB R06-RS202. (D) AF between the E. coli ATCC $11775^{\top}$ genome and all genomes classified as Escherichia in GTDB R06-RS202. Box-and-whisker plots show the lower and upper quartiles as a box, the median value as a line within the box, and the minimum and maximum values as whiskers.

Escherichia coli and Shigella spp. form a single, distinct ANI species cluster

It is informative to consider the clustering which occurs when considering all pairwise ANI values between Escherichia and Shigella genomes and not just the ANI to the type strain/representative genomes of species. Here we explore this using Mash which provides a computationally efficient approximation to ANI allowing it to be applied to large numbers of genomes. Specifically, we use Mash to dereplicate all 23,538 high-quality Escherichia genomes in GTDB R06-R202 to 1,148 genomes which have an ANI <99\% and can be seen as operationally defined strains (see Methods). Visualization of these 1,148 operational strains using a $95 \%$ ANI clustering criterion indicates that $E$. coli, ' $E$. flexneri', ' $E$. dysenteriae', E. coli_C, and E. coli_D form a single cluster in support of reclassifying these 5 GTDB species as a single species (Fig. 3). E. sp005843885 and E. sp004211955 also form a cluster which is unsurprising as the ANI between the GTDB representative genomes of these species is $94.95 \%$.
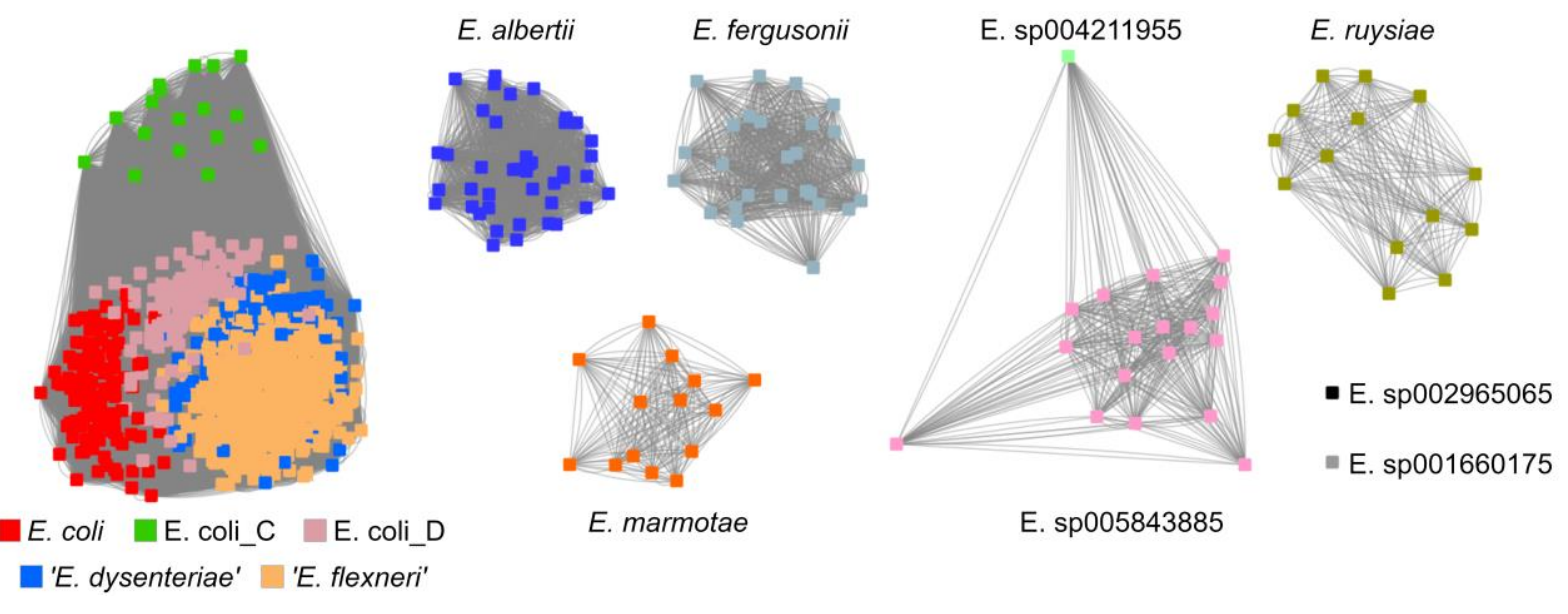

Figure 3. Clustering of 1,148 GTDB R06-RS202 Escherichia genomes dereplicated at $99 \%$ ANI. Each node represents a genome and two genomes are connected by an edge if their Mash distance is $\leq 0.05$, which is approximately equivalent to an ANI $\geq 95 \%$. Nodes are colored by GTDB species assignment. The graph layout was determined using the force directed method implemented in Cytoscape (Shannon et al., 2003).

\section{Conclusions}

In previous releases of GTDB, we attempted to retain at least some Shigella species as distinct entities within the genus Escherichia, i.e. 'E. flexneri' and 'E. dysenteriae', using our type strain nucleated ANIbased species delineation approach (Parks et al., 2020), albeit with reduced ANI radii (Fig. 1A). This was 
a compromise to accommodate the well-known issue of Shigella species being genomically closely related to E. coli (Brenner et al., 1973; Richter and Rosselló-Móra 2009; Jain et al., 2018; Ciufo et al., 2018), while still retaining some of the clinically important classification information associated with Shigella. An unforeseen consequence of this compromise was the reclassification of almost $60 \%$ of $E$. coli genomes including $E$. coli K-12 to 'E. flexneri' (Table 1), which is a source of potential confusion. Indeed, $\mathrm{K}-12$ is often mistakenly thought to be the type strain of $E$. coli, but in fact is genomically and phenotypically quite distinct from the actual type strain, E. coli U5/41 ${ }^{\top}$ (DSM $30083^{\top}$ ), which has been sequenced only comparatively recently (Meier-Kolthoff et al., 2014). To remove this confusion, we intend to treat Shigella species as later heterotypic synonyms of E. coli (Fig. 1B) in the next GTDB release, consistent with previous genomic circumscriptions (Lan and Reeves, 2002; Richter and RossellóMóra, 2009; van den Beld and Reubsaet, 2012), which also returns strain K-12 to the species E. coli. The other major consequence of this change is that the GTDB taxonomy as it currently stands is not useful for clinical classification of Shigella isolates. In our opinion, reclassifying Shigella spp. as later heterotypic synonyms of $E$. coli will ultimately best serve the community as this adheres to the species definition adopted by the GTDB, makes $E$. coli commensurate with the majority of bacterial species, and most accurately reflects the phylogenetic relationship and genomic similarity of E. coli and Shigella strains.

\section{Methods}

\section{Escherichia/Shigella genome dataset}

The 23,686 genomes classified as Escherichia in GTDB R06-RS202 or Escherichia/Shigella according to the NCBI Taxonomy (Schoch et al. 2020; downloaded September 23, 2020) were considered in this study. Two notable exceptions are inclusion of the E. ruysiae type strain genome GCF_902498915.1 which was released after GTDB R06-RS202 and filtering of the genome GCF_009711095.1 which is classified as ' $E$. $a l b a^{\prime}$ ' at NCBI but recognized as belonging to the genus Intestinirhabdus (Xu et al., 2020). The E. ruysiae type strain genome (GCF_902498915.1) was compared to GTDB species representatives in Escherichia and found to be highly similar to the representative of Escherichia sp000208585 (GCF_000208585.1) with an ANI of 99.8\% and AF of 0.97. Consequently, the 32 genomes in the GTDB R06-RS202 species cluster Escherichia sp000208585 are referred to as E. ruysiae in this study. A highquality set of 23,538 genomes defined as having a completeness $\geq 90 \%$ and contamination $\leq 5 \%$ as estimated using CheckM v1.1.3 (Parks et al., 2015) was used for analyses that might be negatively impacted by the presence of lower-quality genome assemblies.

\section{Inferring core gene tree}

Prokka v1.14.6 (Seemann, 2014) using the Escherichia specific database was used to annotate genomes and the core gene set determined using Roary v3.12.0 (Page et al., 2015). The core gene set for the 16 Escherichia/Shigella type strain or GTDB representative genomes was defined as any gene present in $\geq 14$ of the genomes which resulted in a set of 2,329 core genes. These genes were aligned with MAFFT v7.394 (Katoh et al., 2013) which produced a 2,263,396 base multiple sequence alignment. The tree was inferred with IQ-Tree v1.6.12 (Nguyen et al., 2015) using ModelFinder (Kalyaanamoorthy et al., 2017) to establish GTR+F+R5 as the best-fit model and tree stability assessed with the SH-aLRT test and ultrafast bootstraps set to 1,000 replicates (Hoang et al., 2018). The tree was rooted on E. albertii based on a 
preliminary tree using Salmonella enterica $\mathrm{LT}^{\top}$ (GCF_000006945.2) as an outgroup which indicate this Escherchia species to be the most basal member of the genus. This rooting is also in agreement with $E$. albertii being the most basal species in the ANI-based UPGMA tree.

\section{Calculating ANI and AF}

The ANI and AF between genomes was calculated with FastANI v1.3 (Jain et al., 2018) with default parameters unless otherwise indicated. Since the ANI and AF values produced by FastANI are not symmetric, the maximum of the two reciprocal calculations was used in agreement with the approach adopted by GTDB (Parks et al., 2020). ANI values were also calculated with the ani.rb script in the Enveomics Collection (Rodriguez-R LM \& Konstantinidis, 2016) using default parameters and BLASTn 2.9.0+ (Camacho et al., 2009) to identify orthologous fragments.

Mash v2.0 (Ondov et al., 2016) with a sketch size of 5,000 and $k$-mer size of 16 was used to estimate the ANI between all 554,013,906 pairwise combinations of the 23,538 high-quality GTDB R06-RS202 Escherichia genomes as the use of less computationally efficient methods was not practical. Genomes were dereplicated in a greedy manner consisting of three steps: i) sort genomes in descending order of estimated assembly quality, ii) select the highest-quality genome to form a new cluster, and iii) assign any unclustered genome with a Mash distance $<0.01(\approx 99 \% \mathrm{ANI})$ to this new cluster. These steps were repeated until all genomes were assigned to a cluster. Estimated assembly quality was defined as completeness -5 contamination as estimated using CheckM v1.1.3 (Parks et al., 2015).

Pairwise ANI values were visualized as a UPGMA tree calculated with DendroPy v4.5.1 (Sukumaran and Holder, 2010) and as a graph generated with force directed layout method implemented in Cytoscape (Shannon et al., 2003).

\section{Funding}

GTDB is supported by an Australian Research Council Laureate Fellowship (FL150100038) awarded to P.H. and strategic funding from The University of Queensland.

\section{References}

Brenner DJ, et al. 1973. Polynucleotide sequence relatedness among Shigella species. Int J Syst Bacteriol 23: 1-7.

Brenner DJ, et al. 1982. Atypical biogroups of Escherichia coli found in clinical specimens and description of Escherichia hermannii sp. nov. J. Clin. Microbiol. 15: 703-713.

Camacho C, et al. 2009. BLAST+: architecture and applications. BMC Bioinformatics 10: 421.

Castellani A and Chalmers AJ. 1919. Manual of Tropical Medicine, 3rd ed. Williams Wood and Co., New York.

Chattaway MA, et al. 2017. Identification of Escherichia coli and Shigella species from whole-genome sequences. J Clin Microbiol 55: 616-623.

Ciufo S, et al. 2018. Using average nucleotide identity to improve taxonomic assignments in prokaryotic genomes at the NCBI. Int J Syst Evol Microbiol 68: 2386-2392. 
bioRxiv preprint doi: https://doi.org/10.1101/2021.09.22.461432; this version posted September 22, 2021. The copyright holder for this preprint (which was not certified by peer review) is the author/funder, who has granted bioRxiv a license to display the preprint in perpetuity. It is made available under aCC-BY 4.0 International license.

Doolittle WF and Papke RT. Genomics and the bacterial species problem. Genome Biology 7: 116.

Ewing WH, 1949. Shigella nomenclature. J Bacterio/ 57: 633-638.

Farmer JJ, et al., 1985. Escherichia fergusonii and Enterobacter taylorae, two new species of Enterobacteriaceae isolated from clinical specimens. J clin Microbiol 21: 77-81.

Goris J, et al. 2007. DNA-DNA hybridization values and their relationship to whole-genome sequence similarities. Int J Syst Evol Microbiol 57: 81-91.

Hata $\mathrm{H}$, et al. 2016. Phylogenetics of family Enterobacteriaceae and proposal to reclassify Escherichia hermannii and Salmonella subterranea as Atlantibacter hermannii and Atlantibacter subterranea gen. nov., comb. nov. Microbiol Immunol 60: 303311.

Hendriks ACA, et al. 2020. Genome-wide association studies of Shigella spp. and enteroinvasive Escherichia coli isolates demonstrate an absence of genetic markers for prediction of disease severity. BMC Genomics 21: 138.

Hoang DT, et al. 2018. UFBoot2: Improving the ultrafast bootstrap approximation. Mol Biol Evol 35: 518-522.

Hu D, et al. 2019. Living Trees: high-quality reproducible and reusable construction of bacterial phylogenetic trees. Mol Biol Evol 37: 563-575.

Huys G, et al. 2003. Escherichia albertii sp. nov., a diarrhoeagenic species isolated from stool specimens of Bangladeshi children. Int J Syst Evol Microbiol 53: 807-810.

Jain C, et al. 2018. High throughput ANI analysis of 90K prokaryotic genomes reveals clear species boundaries. Nat Commun 9 : 5114.

Johnson JR. 2000. Shigella and Escherichia coli at the crossroads: Machiavellian masqueraders or taxonomic treachery? J Med Microbiol 49: 583-585.

Katoh K, Standley DM. 2013. MAFFT multiple sequence alignment software version 7: improvements in performance and usability. Mol Biol Evol 30: 772-780.

Kalyaanamoorthy S, et al. 2017. ModelFinder: Fast model selection for accurate phylogenetic estimates. Nat Methods 14: 587589.

Konstantinidis KT and Tiedje JM. 2005. Genomic insights that advance the species definition for prokaryotes. Proc Natl Acad Sci U S A 102: 2567-72.

Konstantinidis KT, et al. 2006. The bacterial species definition in the genomic era. Philos Trans R Soc Lond B Biol Sci 361: 192940.

Lan R and Reeves PR, 2002. Escherichia coli in disguise: molecular origins of Shigella. Microbes Infect. 4: 1125-32.

Liu S, et al. 2015. Escherichia marmotae sp. nov., isolated from faeces of Marmota himalayana. Int J Syst Evol Microbiol 65: 2130-2134.

Meier-Kolthoff JP, et al. 2014. Complete genome sequence of DSM 30083 ${ }^{\top}$, the type strain (U5/41 ${ }^{\top}$ ) of Escherichia coli, and a proposal for delineating subspecies in microbial taxonomy. Stand in Genomic Sci 9: https://doi.org/10.1186/1944-3277-9-2.

Minh BQ, et al. 2020 IQ-TREE 2: New models and efficient methods for phylogenetic inference in the genomic era. Mol Biol Evol, 37: 1530-1534.

Nguyen L-T, et al. 2015. IQ-TREE: A fast and effective stochastic algorithm for estimating maximum likelihood phylogenies. Mol Biol Evol, 32: 268-274.

Ondov BD, et al. 2016. Mash: fast genome and metagenome distance estimation using MinHash. Genome Biology 17: 132.

Page AJ, et al. 2015. Roary: rapid large-scale prokaryote pan genome analysis. Bioinformatics 31: 3691-3. 
Parks DH, et al. 2015. CheckM: assessing the quality of microbial genomes recovered from isolates, single cells, and metagenomes. Genome Res 25: 1043-1055.

Parks DH, et al. 2018. A standardized bacterial taxonomy based on genome phylogeny substantially revises the tree of life. Nat Biotechnol 36: 996-1004.

Parks DH, et al. 2020. A complete domain-to-species taxonomy for Bacteria and Archaea. Nat Biotechnol 38: $1079-1086$.

Pattengill EA, et al. 2016. Phylogenetic analyses of Shigella and enteroinvasive Escherichia coli for the identification of molecular epidemiological markers: whole-genome comparative analysis does not support distinct genera designation. Front Microbiol 6: 1573.

Pupo GM, et al. 2000. Multiple independent origins of Shigella clones of Escherichia coli and convergent evolution of many of their characteristics. Proc Natl Acad Sci USA 97: 10567-10572.

Richter M and Rosselló-Móra R. 2009. Shifting the genomic gold standard for the prokaryotic species definition. Proc Natl Acad Sci USA 106: 19126-19131.

Rodriguez-R LM and Konstantinidis KT. 2016. The enveomics collection: a toolbox for specialized analyses of microbial genomes and metagenomes. PeerJ Preprints 4: e1900v1.

Sahl JW, et al. 2015. Defining the phylogenomics of Shigella species: a pathway to diagnostics. J Clin Microbiol 53: 951-60.

Schoch et al., 2020. NCBI Taxonomy: a comprehensive update on curation, resources and tools. Database (Oxford), doi: 10.1093/database/baaa062.

Seemann T. 2014. Prokka: rapid prokaryotic genome annotation. Bioinformatics 30: 2068-2069.

Shannon P, et al. 2003. Cytoscape: a software environment for integrated models of biomolecular interaction networks. Genome Res 13: 2498-504.

Sukumaran J and Holder MT. 2010. DendroPy: A Python library for phylogenetic computing. Bioinformatics 26: $1569-1571$.

The HC, et al. 2016. The genomic signatures of Shigella evolution, adaptation and geographical spread. Nat Rev Microbiol 14: 235-250.

van den Beld MJ and Reubsaet FA. 2012. Differentiation between Shigella, enteroinvasive Escherichia coli (EIEC) and noninvasive Escherichia coli. Eur J Clin Microbiol Infect Dis 31: 899-904.

van der Putten BCL, et al., 2021. Escherichia ruysiae sp. nov., a novel Gram-stain-negative bacterium, isolated from a faecal sample of an international traveller. Int J Syst Evol Microbiol 71: doi: 10.1099/ijsem.0.004609.

Varghese NJ, et al. 2015. Microbial species delineation using whole genome sequences. Nucleic Acids Res 43: 6761-71.

Walk ST, et al. 2009. Cryptic lineages of the genus Escherichia. Appl Environ Microbiol 75: 6534-6544.

$\mathrm{Xu}$ Z, et al. 2020. Intestinirhabdus alba gen. nov., sp. nov., a novel genus of the family Enterobacteriaceae, isolated from the gut of plastic-eating larvae of the Coleoptera insect Zophobas atratus. Int J Syst Evol, 70: https://doi.org/10.1099/ijsem.0.004364.

Yang F, et al. 2005. Genome dynamics and diversity of Shigella species, the etiologic agents of bacillary dysentery. Nucleic Acids Res. 33: 6445-6458. 


\section{Supplementary Figures and Tables}
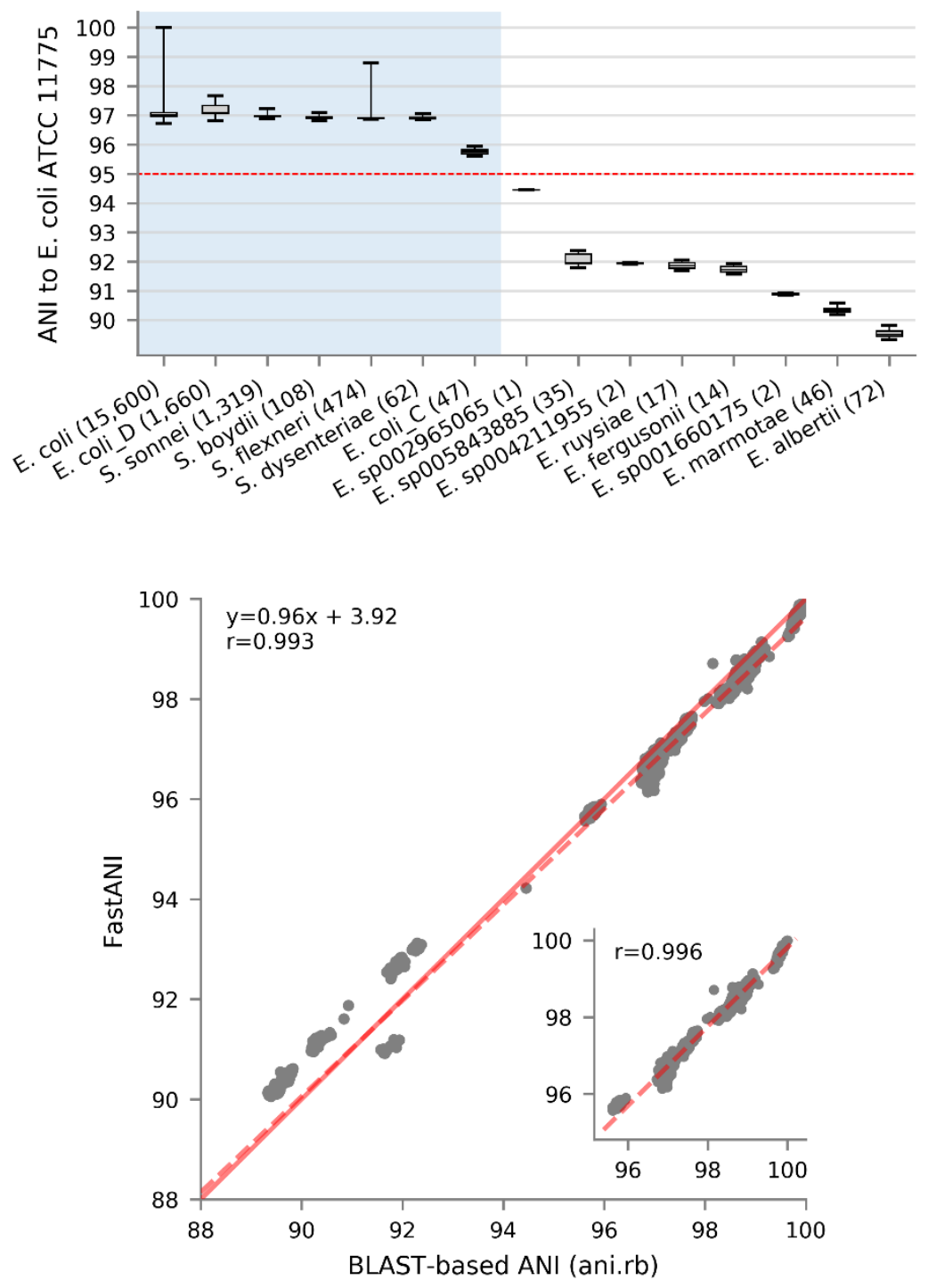

Supplementary Figure 1. ANI between the E. coli ATCC $11775^{\top}$ genome and all genomes classified as Escherichia in GTDB R06RS202. (A) ANI values calculated with the BLAST-based ani.rb script in the Enveomics Collection. This plot is analogous to the FastANI results provided in Figure 2C. (B) Correlation between ANI values calculated with FastANI and ani.rb. The $y=x$ line is shown in solid red, the best-fit line is shown as red dashes, and the Pearson correlation coefficients is given in the upper left corner. The inset plot shows results for E. coli, 'E. flexneri', 'E. dysenteriae', E. coli_C, and E. coli_D genomes.

Supplementary Table 1. Incongruent species classification between GTDB and NCBI (see Excel file). 
bioRxiv preprint doi: https://doi.org/10.1101/2021.09.22.461432; this version posted September 22, 2021. The copyright holder for this preprint (which was not certified by peer review) is the author/funder, who has granted bioRxiv a license to display the preprint in perpetuity. It is made available under aCC-BY 4.0 International license.

Supplementary Table 2. NCBI species with the largest number of reassignments in GTDB.

\begin{tabular}{|c|c|c|c|c|}
\hline NCBI species & $\begin{array}{l}\text { No. } \\
\text { genomes }\end{array}$ & $\begin{array}{l}\text { No. } \\
\text { reassigned } \\
\text { genomes }\end{array}$ & $\begin{array}{l}\text { Reassigned } \\
\text { genomes } \\
(\%)\end{array}$ & GTDB species \\
\hline Escherichia coli & 20,973 & $\begin{array}{c}16,609 \\
(79.19 \%)\end{array}$ & 28.28 & 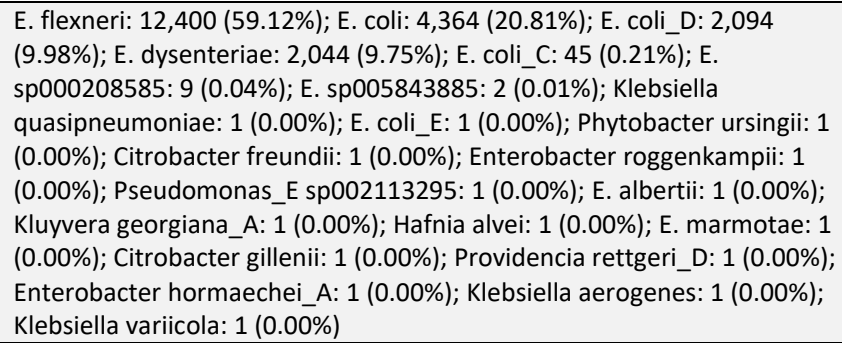 \\
\hline Enterococcus faecium & 2,019 & $\begin{array}{c}2,019 \\
(100.00 \%)\end{array}$ & 3.44 & $\begin{array}{l}\text { Enterococcus_B faecium: 1,793 (88.81\%); Enterococcus_B lactis: } 224 \\
\text { (11.09\%); Enterococcus_D sp002850555: } 2 \text { (0.10\%) }\end{array}$ \\
\hline Listeria monocytogenes & 3,525 & $\begin{array}{c}1,740 \\
(49.36 \%)\end{array}$ & 2.96 & $\begin{array}{l}\text { L. monocytogenes: 1,785 (50.64\%); L.monocytogenes_B: 1,562 (44.31\%); } \\
\text { L. monocytogenes_C: } 172(4.88 \%) \text {; L. innocua: } 6(0.17 \%)\end{array}$ \\
\hline Mycobacteroides abscessus & 1,686 & $\begin{array}{c}1,686 \\
(100.00 \%)\end{array}$ & 2.87 & Mycobacterium abscessus: 1,686 (100.00\%) \\
\hline Campylobacter jejuni & 1,678 & $\begin{array}{c}1,678 \\
(100.00 \%)\end{array}$ & 2.86 & $\begin{array}{l}\text { Campylobacter_D jejuni: } 1,670 \text { (99.52\%); Campylobacter_D lari_C: } 3 \\
\text { (0.18\%); Campylobacter_D jejuni_D: } 2 \text { (0.12\%); Campylobacter_D } \\
\text { jejuni_C: } 1(0.06 \%) \text {; Campylobacter_D jejuni_B: } 1(0.06 \%) ; \\
\text { Campylobacter_D jejuni_A: } 1(0.06 \%)\end{array}$ \\
\hline Burkholderia pseudomallei & 1,604 & $\begin{array}{c}1,604 \\
(100.00 \%)\end{array}$ & 2.73 & Burkholderia mallei: 1,604 (100.00\%) \\
\hline Pseudomonas viridiflava & 1,518 & $\begin{array}{c}1,518 \\
(100.00 \%)\end{array}$ & 2.58 & 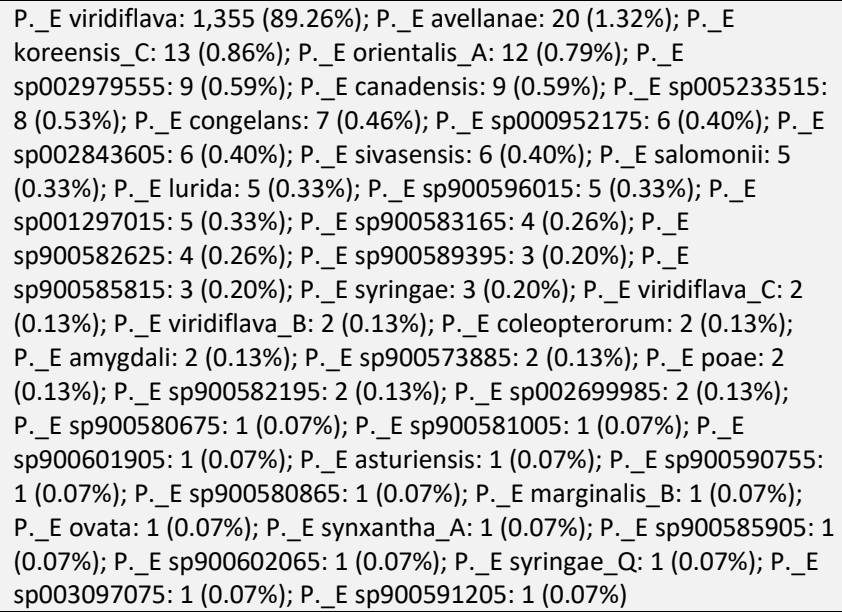 \\
\hline Enterobacter hormaechei & 1,458 & $\begin{array}{c}1,444 \\
(99.04 \%)\end{array}$ & 2.46 & $\begin{array}{l}\text { E. hormaechei_A: } 1,440 \text { (98.77\%); E. hormaechei: } 14(0.96 \%) ; \text { E. } \\
\text { chengduensis: } 1(0.07 \%) \text {; Escherichia coli: } 1(0.07 \%) ; \text { E. asburiae_B: } 1 \\
(0.07 \%) ; \text { E. quasihormaechei: } 1(0.07 \%)\end{array}$ \\
\hline Shigella sonnei & 1,368 & $\begin{array}{c}1,368 \\
(100.00 \%)\end{array}$ & 2.33 & $\begin{array}{l}\text { E. flexneri: 1,354 (98.98\%); E. coli_D: } 13(0.95 \%) \text {; Serratia marcescens_K: } \\
1(0.07 \%)\end{array}$ \\
\hline Bacillus cereus & 1,094 & $\begin{array}{c}1,094 \\
(100.00 \%)\end{array}$ & 1.86 & 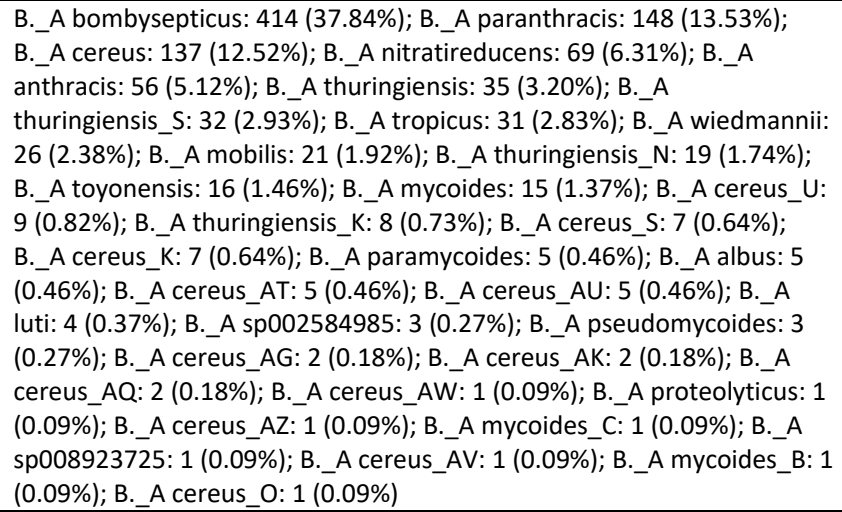 \\
\hline
\end{tabular}


bioRxiv preprint doi: https://doi.org/10.1101/2021.09.22.461432; this version posted September 22, 2021. The copyright holder for this preprint (which was not certified by peer review) is the author/funder, who has granted bioRxiv a license to display the preprint in perpetuity. It is made available under aCC-BY 4.0 International license.

Supplementary Table 3. Genomes classified as an Escherichia or Shigella species at NCBI which are misclassified based on their ANI to the type strain of the species.

\begin{tabular}{|c|c|c|c|c|}
\hline Genome ID & NCBI species & ANI to type strain genome & AF to type strain genome & GTDB species \\
\hline GCF_000601195.1 & Escherichia coli & 92.85 & 0.87 & Escherichia sp000208585 \\
\hline GCF_903932005.1 & Escherichia coli & 92.85 & 0.83 & Escherichia sp005843885 \\
\hline GCF_003145355.1 & Escherichia coli & 92.84 & 0.87 & Escherichia sp000208585 \\
\hline GCA_013425105.1 & Escherichia coli & 92.83 & 0.86 & Escherichia sp000208585 \\
\hline GCF_005400045.1 & Escherichia coli & 92.77 & 0.85 & Escherichia sp000208585 \\
\hline GCF_002110245.1 & Escherichia coli & 92.7 & 0.86 & Escherichia sp000208585 \\
\hline GCF_004745245.1 & Escherichia coli & 92.65 & 0.81 & Escherichia sp005843885 \\
\hline GCF_000459855.1 & Escherichia coli & 92.58 & 0.84 & Escherichia sp000208585 \\
\hline GCF_903932105.1 & Escherichia coli & 92.52 & 0.88 & Escherichia sp000208585 \\
\hline GCF_000398885.1 & Escherichia coli & 92.47 & 0.85 & Escherichia sp000208585 \\
\hline GCA_001630835.1 & Escherichia coli & 92.43 & 0.85 & Escherichia sp000208585 \\
\hline GCF_011881725.1 & Escherichia coli & 92.22 & 0.89 & Escherichia coli_E \\
\hline GCF_903932125.1 & Escherichia coli & 91.09 & 0.81 & Escherichia marmotae \\
\hline GCF_001286085.1 & Escherichia coli & 90.28 & 0.8 & Escherichia albertii \\
\hline GCF_900448175.1 & Escherichia coli & 82.35 & 0.57 & Citrobacter gillenii \\
\hline GCF_003007795.1 & Escherichia coli & 82.23 & 0.57 & Citrobacter freundii \\
\hline GCA_011008595.1 & Escherichia coli & 81.4 & 0.46 & Kluyvera georgiana_A \\
\hline GCA_009766545.1 & Escherichia coli & 81.39 & 0.51 & Enterobacter hormaechei_A \\
\hline GCF_006381975.1 & Escherichia coli & 81.33 & 0.5 & Enterobacter roggenkampii \\
\hline GCA_003175335.1 & Escherichia coli & 81.19 & 0.44 & Klebsiella variicola \\
\hline GCA_003301495.1 & Escherichia coli & 81.05 & 0.44 & Klebsiella aerogenes \\
\hline GCA_003363055.1 & Escherichia coli & 80.98 & 0.44 & Phytobacter ursingii \\
\hline GCA_003176195.1 & Escherichia coli & 80.9 & 0.42 & Klebsiella quasipneumoniae \\
\hline GCF_009647995.1 & Escherichia coli & $0^{*}$ & $0 *$ & Pseudomonas_E sp002113295 \\
\hline GCF_011008745.1 & Escherichia coli & $0 *$ & 0* & Hafnia alvei \\
\hline GCF_009648115.1 & Escherichia coli & $0 *$ & $0^{*}$ & Providencia rettgeri_D \\
\hline GCF_902167795.1 & Escherichia fergusonii & 91.03 & 0.69 & 'Escherichia flexneri' \\
\hline GCF_001060475.1 & Shigella boydii & 78.85 & 0.25 & Serratia marcescens_I \\
\hline GCF_001062045.1 & Shigella boydii & 78.82 & 0.23 & Serratia marcescens_I \\
\hline GCF_001060695.1 & Shigella boydii & 78.73 & 0.23 & Serratia marcescens_I \\
\hline GCA_001066285.1 & Shigella sonnei & 78.85 & 0.24 & Serratia marcescens_K \\
\hline
\end{tabular}

${ }^{*}$ sufficiently divergent that FastANI fails to provide an ANI or AF value

Supplementary Table 4. Type strain and GTDB representative genomes.

\begin{tabular}{cllccc} 
Genome ID & GTDB species & NCBI species & Strain ID & $\begin{array}{c}\text { GTDB } \\
\text { representative }\end{array}$ & $\begin{array}{c}\text { Type strain } \\
\text { of species }\end{array}$ \\
\hline GCF_001660175.1 & Escherichia sp001660175 & unclassified & B1147 & TRUE & FALSE \\
\hline GCF_004211955.1 & Escherichia sp004211955 & unclassified & E1V33 & TRUE & FALSE \\
\hline GCF_002965065.1 & Escherichia sp002965065 & unclassified & MOD1-EC7003 & TRUE & FALSE \\
\hline GCF_005843885.1 & Escherichia sp005843885 & unclassified & E4742 & TRUE & FALSE \\
\hline GCF_000759775.1 & Escherichia albertii & Escherichia albertii & NBRC 107761 & TRUE & TRUE \\
\hline GCF_011881725.1 & Escherichia coli_E & Escherichia coli & SCPM-O-B-8794 & TRUE & FALSE \\
\hline GCF_000026325.1 & Escherichia coli_D & Escherichia coli & UMN026 & TRUE & FALSE \\
\hline GCA_003018335.1 & Escherichia coli_C & Escherichia coli & 2013C-4282 & TRUE & FALSE \\
\hline GCF_003697165.2 & Escherichia coli & Escherichia coli & ATCC 11775 & TRUE & TRUE \\
\hline GCF_000026225.1 & Escherichia fergusonii & Escherichia fergusonii & ATCC 35469 & TRUE & TRUE \\
\hline GCF_002900365.1 & Escherichia marmotae & Escherichia marmotae & HT073016 & TRUE & TRUE \\
\hline GCF_902498915.1 & Escherichia ruysiae & Escherichia ruysiae & OPT1704 & n/a* & TRUE \\
\hline GCF_002946735.1 & 'Escherichia flexneri' & Shigella boydii & ATCC 8700 & FALSE & TRUE \\
\hline GCF_002949675.1 & 'Escherichia dysenteriae' & Shigella dysenteriae & ATCC 13313 & TRUE & TRUE \\
\hline GCF_002950215.1 & 'Escherichia flexneri' & Shigella flexneri & ATCC 29903 & TRUE & TRUE \\
\hline GCA_002950395.1 & 'Escherichia flexneri' & Shigella sonnei & ATCC 29930 & FALSE & TRUE \\
\hline * released after GTDB R06-RS202 & & & &
\end{tabular}


bioRxiv preprint doi: https://doi.org/10.1101/2021.09.22.461432; this version posted September 22, 2021. The copyright holder for this preprint (which was not certified by peer review) is the author/funder, who has granted bioRxiv a license to display the preprint in perpetuity. It is made available under aCC-BY 4.0 International license.

Supplementary Table 5. ANI (lower triangle) and AF (upper triangle) between type strain genomes from E. coli and Shigella species and GTDB representative genomes for E. coli_C and E. coli_D.

\begin{tabular}{|c|c|c|c|c|c|c|c|}
\hline & E. coli & E. coli_C & E. coli_D & S. boydii & S. dysenteriae & S. flexneri & S. sonnei \\
\hline \multirow{2}{*}{$\begin{array}{r}\text { E. coli } \\
\text { E. coli_C }\end{array}$} & - & 0.84 & 0.86 & 0.80 & 0.78 & 0.78 & 0.79 \\
\hline & 95.64 & - & 0.88 & 0.83 & 0.81 & 0.80 & 0.81 \\
\hline \multirow{2}{*}{$\begin{array}{l}\text { E. coli_D } \\
\text { S. boydii }\end{array}$} & 96.81 & 96.13 & - & 0.83 & 0.81 & 0.80 & 0.81 \\
\hline & 96.47 & 95.45 & 96.95 & - & 0.78 & 0.84 & 0.86 \\
\hline \multirow{3}{*}{$\begin{array}{r}\text { S. dysenteriae } \\
\text { S. flexneri } \\
\text { S. sonnei }\end{array}$} & 96.30 & 95.27 & 96.65 & 97.06 & - & 0.81 & 0.80 \\
\hline & 96.40 & 95.61 & 96.95 & 97.81 & 97.00 & - & 0.80 \\
\hline & 96.55 & 95.59 & 97.02 & 98.14 & 97.15 & 97.94 & - \\
\hline
\end{tabular}

